11

\title{
Частотно-селективное распространение спиновых волн в трехмерном магнонном Т-образном сплиттере
}

\author{
(C) А.А. Мартышкин, Е.Н. Бегинин, А.В. Садовников \\ Саратовский государственные университет им. Н.Г. Чернышевского, \\ 410012Саратов, Россия \\ e-mail: aamartyshkin@gmail.com
}

Поступило в Редакцию 30 апреля 2021 г.

В окончательной редакции 30 апреля 2021 г.

Принята к публикации 30 апреля 2021 г.

С помощью численных и экспериментальных методов исследован механизм управления передачи спин-волнового сигнала в трехмерном магнонном сплиттере, образованном ортогональным сочленением магнитных полосок железо-иттриевого граната. Показано, что при изменении величины воздушного зазора между спин-волноведущими секциями оказывается возможным управлять селекцией сигнала, распространяющегося в выходных секциях структуры. С прикладной точки зрения, полученные результаты могут быть использованы для создания элемента межсоединения в многоуровневых магнонных устройствах обработки информации для формирования многослойных топологий магнонных сетей и миниатюризации вычислительных устройств, основанных на принципах магноники.

Ключевые слова: спиновые волны, магноника, трехмерные межсоединения, микромагнитное моделирование.

DOI: $10.21883 / J T F .2021 .10 .51370 .134-21$

\section{Введение}

В настоящее время активно изучаются механизмы и методы возбуждения и управления спиновыми волнами в магнитных материалах $[1,2]$ в рамках научных направлений физики конденсированного состояния таким как магноника [3-5]. Использование передачи сигнала без движения зарядов в виде элементарных квантов магнитных возбуждений (магнонов) и спиновых волн (CB) в диэлектрических магнитных пленках является многообещающей альтернативой полупроводниковым приборам, обеспечивая сверхнизкое энергопотребление из-за отсутствия рассеяния энергии на омические потери [6-8]. Совершенствование технологического процесса изготовления тонких пленок способствует созданию микро- и наноструктур, объединение которых в многоуровневые схемы позволяет создавать магнонные сети (MC) [9,10], принцип действия которых основан на эффектах интерференции и позволяет кодировать сигнал с помощью амплитуды и фазы спиновой волны $[10,11]$.

Большинство схем, основанных на магнонной логике, намагничены в плоскости, что накладывает ограничения на маршрутизацию сигналов, поскольку магнонные сети ограничены одним функциональным уровнем, имеют критическую длину распространения сигнала и большую площадь устройства [12]. Изготовление структур с вертикальным транспортом спин-волнового сигнала позволяет создавать трехмерные магнонные сети с большим количеством функциональных блоков в меньшем объеме. На пути к увеличению количества функциональных элементов в МС оказывается важным изучить механизмы, ответственные за перенос СВ в многоуровневых топологиях МС на основе трехмерных структур $[13,14]$.

Недавно концепция использования трехмерной магнонной структуры была продемонстрирована в магнонном кристалле, изготовленном в виде меандровых пленок $\mathrm{CoFeB}$ [15] и железо-итриевых гранатов (ЖИГ или YIG) [16], состоящих из ферромагнитных сегментов, расположенных под углом 90 друг к другу. Такая геометрия имеет преимущество перед обычными магнонными кристаллами, позволяя избежать ограничения, связанные с транспортом и управлением CB в плоских намагниченных пленках из-за анизотропной дисперсии и позволяя распространять СВ в трех измерениях без значительных потерь в области перехода.

В настоящей работе предлагается и исследуется вариант реализации межуровневого транспорта СВ между параллельными слоями МС на основе ортогонально соединенных магнитных СВЧ волноводов. Предлагаемый функциональный элемент соединения магнонных уровней позволяет реализовать селекцию частот, что может быть использовано при реализации алгоритмов обработки сигналов. С помощью численных и экспериментальных исследований продемонстрирован механизм спин-волнового транспорта в системе из двух латеральных магнитных полосок, сочлененных ортогонально. Показана эффективная селекция спин-волнового сигнала с помощью изменения воздушного зазора в области сочленения волноведущих магнитных полосок. 


\section{1. Структура и численное исследование}

Для исследования механизмов управления спинволновой связью было проведено микромагнитное моделирование в программе MuMax3 [17] на основе численного решения уравнения Ландау-Лифшица-Гильберта:

$$
\frac{\partial \mathbf{M}}{\partial t}=-\gamma\left[\mathbf{H}_{\mathrm{eff}} \times \mathbf{M}\right]+\frac{\alpha}{M_{s}}\left[\mathbf{M} \times \frac{\partial \mathbf{M}}{\partial t}\right],
$$

которое описывает прецессию магнитного момента М в эффективном магнитном поле $\mathbf{H}_{\text {eff }}=\mathbf{H}_{0}+\mathbf{H}_{\text {demag }}$ $+\mathbf{H}_{\mathrm{ex}}+\mathbf{H}_{\mathrm{a}}$, где $\mathbf{H}_{0}-$ внешнее магнитное поле, $\mathbf{H}_{\text {demag }}$ - поле размагничивания, $\mathbf{H}_{\mathrm{ex}}$ - обменное поле, $\mathbf{H}_{\mathrm{a}}$ - поле анизотропии. При этом поле анизотропии полагалось равным $\mathbf{H}_{\mathrm{a}}=0$, поскольку вектор равновесной намагниченности направлен вдоль осей симметрии ЖИГ [111]. Для уменьшения отражений сигнала от границ расчетной области были введены регионы с увеличивающимся в геометрической прогрессии коэффициентом затухания $\alpha=10^{-5}-1$ на границах волноведущей структуры [18]. Для создания структуры в качестве магнитных микроволноводов использовались тонкие пленки ЖИГ $\left[\mathrm{Y}_{3} \mathrm{Fe}_{2}\left(\mathrm{FeO}_{4}\right)_{3}\right.$ (111)] толщиной $10 \mu \mathrm{m}$ и намагниченностью насыщения $4 \pi \mathrm{Ms}=1750 \mathrm{G}$. Безразмерный параметр диссипации полагался равным $\alpha=10^{-5}$, а обменная жесткость $A_{\mathrm{ex}}=3 \cdot 10^{-7} \mathrm{erg} / \mathrm{cm}$. Исследуемая структура может быть представлена в виде Т-образной волноведущей системы, представляющая собой две магнитные полоски, образующих три спин-волновых канала длинной $\mathrm{S}_{1}=\mathrm{S}_{2}=\mathrm{S}_{2}=1280 \mu \mathrm{m}$, помещенных во внешнее однородное магнитное поле $\mathbf{H}_{0}=370$ Ое, направленное вдоль оси $y$ (рис. 1,a). Распределение намагниченности $\mathbf{H}_{\text {int }}=\mathbf{H}_{0}+\mathbf{H}_{\text {demag, }}$, полученное из решения статической задачи в сечении волноведущих секций $\mathrm{S}_{1,3}$ и $\mathrm{S}_{2}$, представлено на рис. $1, b$ и $c$ соответственно. Видно, что внутреннее поле $\mathbf{H}_{\text {int }}$ практически совпадает по величине с $\mathbf{H}_{0}$, что обеспечивает эффективное возбуждение поверхностных магнитостатических волн (ПМСВ) во всей структуре.

Был проведен расчет спектральной плотности мощности выходного сигнала в областях, обозначенных микрополосковыми антеннами $\left(\mathrm{P}_{1,2,3}\right)$ на рис. 1. При этом входной сигнал переменного магнитного поля, создаваемого микрополоском с током, задавался в виде $b_{z}(t)=b_{0} \sin c\left(2 \pi f_{c} t\right)$, где $f_{c}=10 \mathrm{GHz}, b_{0}=10 \mathrm{mOe}$. Значения динамической намагниченности $m_{z}(x, y, t)$ записывались с шагом $\delta t=75 \mathrm{fs}$ в течение времени $T=300 \mathrm{~ns}$. Далее, используя фурье-преобразование, были построены частотные зависимости динамической намагниченности $\mathrm{P}_{2}(f), \mathrm{P}_{3}(f)$ для ширин воздушного зазора $d=0,10,50,200 \mu \mathrm{m}$ при возбуждении спин-волнового сигнала в порте $\mathrm{P}_{1}$ (рис. 2). Детектирование сигнала в области порта $\mathrm{P}_{2}$ демонстрирует спектр

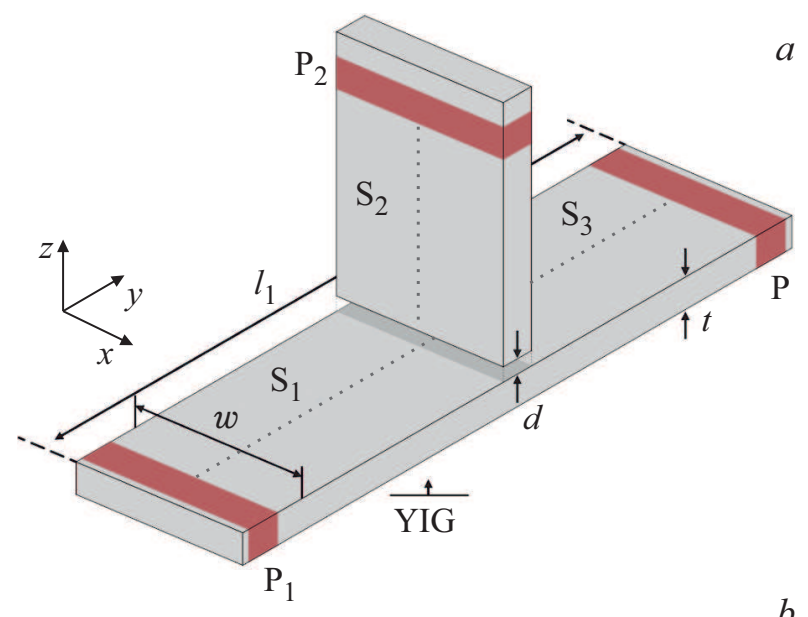

$a$
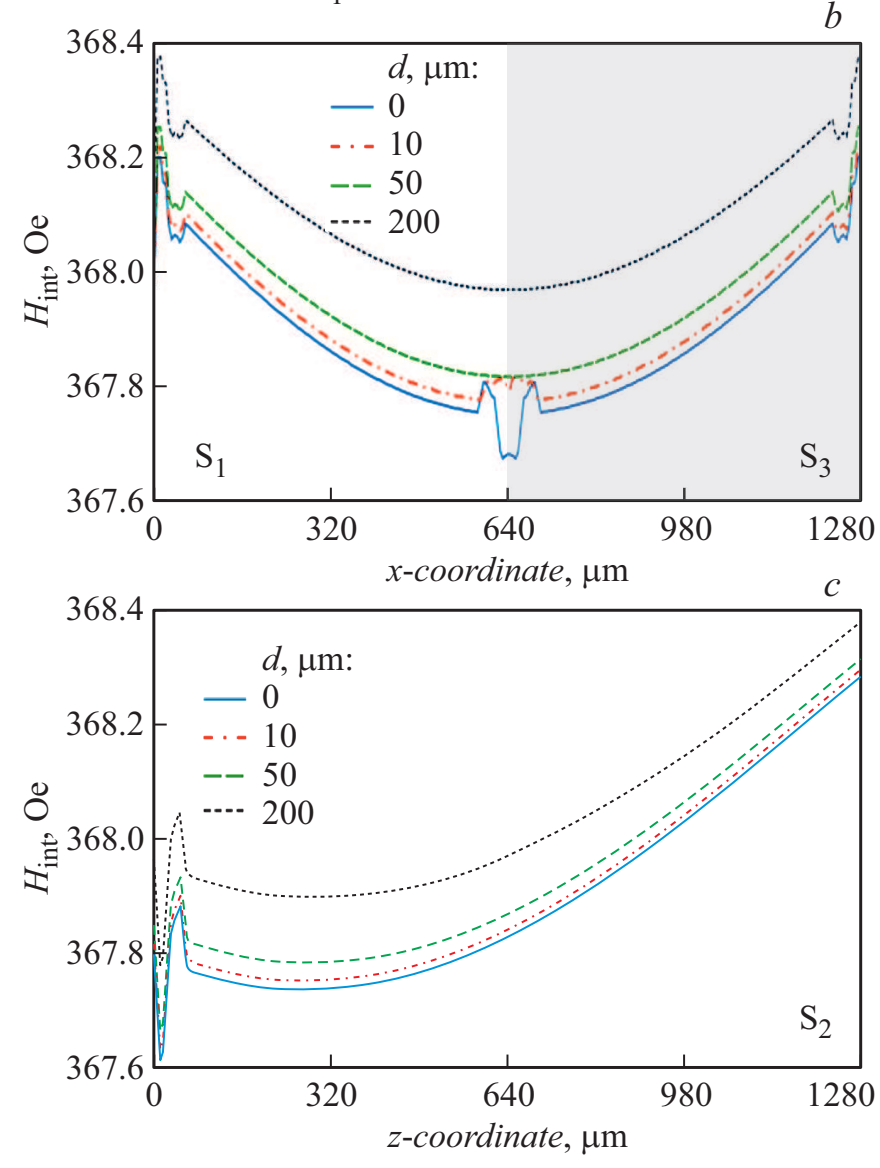

Рис. 1. Схематическое изображение исследуемой структуры $(a)$. Распределение внутреннего магнитного поля $H_{\text {int }}$ в структуре с зазором в области сочленения $d=0,10,20$, $50,200 \mu \mathrm{m}(b)$ в центральном сечении волновода $\mathrm{S}_{1,3},(c)$ в центральном сечении волновода $\mathrm{S}_{2}$.

ПМСВ с провалами, связанными с поворотом спинволнового сигнала в области сочленения секции $\mathrm{S}_{2}$ (рис. 2,a). Уменьшение воздушного зазора между секциями увеличивает амиплитуду детектируемого на порте $\mathrm{P}_{2}$ сигнала. Сигнал, полученный на порте $\mathrm{P}_{3}$, соответствует спектру ПМСВ с искажениями, связанными с затуханием CB. 

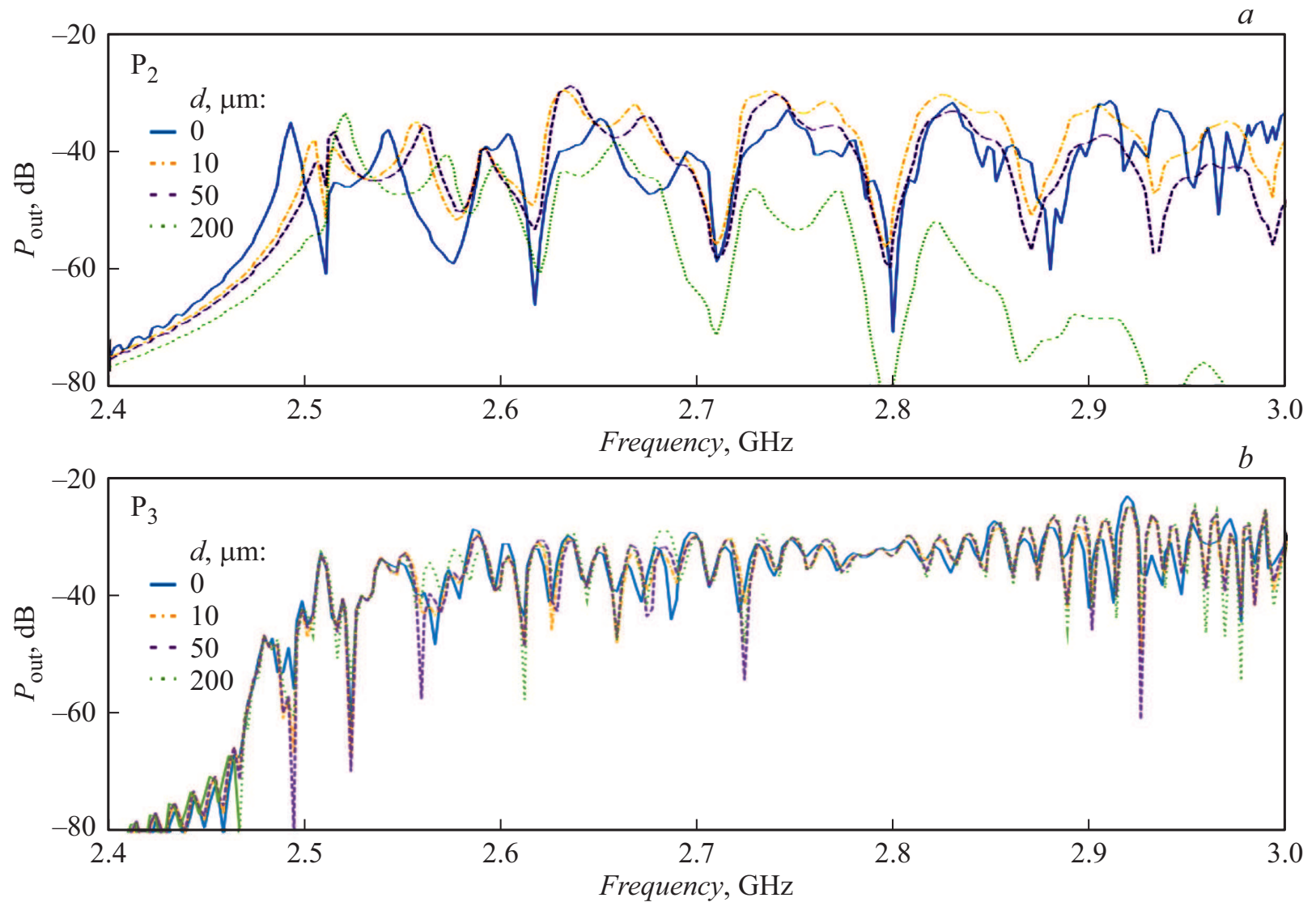

Рис. 2. Частотный спектр спин-волнового сигнала в детектированный в области $(a) \mathrm{P}_{2}$ и $(b) \mathrm{P}_{3}$ в зависимости от величины воздушного зазора.

\section{2. Экспериментальное исследование}

Методом микроволновой спектроскопии было проведено экспериментальное исследование спин-волнового транспорта в изготовленной структуре путем использования микрополосковой линии передачи. С помощью метода лазерного скрайбирования из пленки железо-иттриевого граната были изготовлены магнитные полоски шириной $w=500 \mu \mathrm{m}$ и толщиной $t=10 \mu \mathrm{m}$ на подложке из галлий-гадолиниевого граната $\left[(\mathrm{GGG}) \mathrm{Gd}_{3} \mathrm{Ga}_{5} \mathrm{O}_{12}(111)\right]$ толщиной $500 \mu \mathrm{m}$. Длина горизонтальных секций в экспериментальном исследовании составляла $\mathrm{S}_{1}=\mathrm{S}_{2}=\mathrm{S}_{2}=3000 \mu \mathrm{m}$. Возбуждение CВ осуществлялось с помощью микрополосковой антенны толщиной $1 \mu \mathrm{m}$ и шириной $30 \mu \mathrm{m}$. Структура помещена во внешнее статическое магнитное поле, $H_{0}=370$ Ое, ориентированное вдоль оси х для эффективного возбуждения ПМСВ.

При этом было проведено измерение S-параметров на векторном анализаторе цепей Agilent Technologies PNA Network Analyzer E8362C. Результаты измерения приведены на рис. 3, где показаны частотная зависимость параметров $\mathrm{S}_{n m}$, соответствующих приему сигнала на микрополосковых преобразователях $\mathrm{P}_{n}(n=1,2,3)$ при возбуждении СВЧ сигнала на одном из микрополосков
$\mathrm{P}_{m}(m=1,2,3)$. На рис. $3, a, b$ показана частотная зависимость модуля коэффициентов $\mathrm{S}_{31}$ и $\mathrm{S}_{13}$, соответствующих возбуждению сигнала в порте $\mathrm{P}_{1}$ и $\mathrm{P}_{3}$ и детектированию сигнала на порте $\mathrm{P}_{3}$ и $\mathrm{P}_{1}$ соответственно. Видно, что при генерации сигнала в порте $\mathrm{P}_{3}$ спектральная характеристика соответствует ПМСВ и сигнал выше на $15 \mathrm{~dB}$, чем при генерации сигнала на $\mathrm{P}_{1}$. Такое падение сигнала связано с распространением сигнала по стороне магнитной полоски, к которой присоединена вертикальная секция $\mathrm{S}_{2}$. Возбуждение $\mathrm{CB}$ сигнала на портах $\mathrm{P}_{2}$ и $\mathrm{P}_{1}$ при детекции на портах $\mathrm{P}_{1}$ и $\mathrm{P}_{2}$ соответственно позволяет получать сигнал амплитудой до $-30 \mathrm{~dB}$.

На рис. $3, c, d$ показан результат расчета волновых чисел в диапазоне частот возбуждения ПМСВ $k=\psi / L$, где $\psi$ - фазовый сдвиг $\mathrm{CB}$, который происходит на длине $L$ между входом $\mathrm{P}_{n}$ и выход $\mathrm{P}_{m}$ в секции. Видно, что в секции $\mathrm{S}_{13}$ возбуждаемый тип волны (ПМСВ) приводит к зависимости $f(k)$, которая качественно совпадает с дисперсионной зависимостью для мод ПМСВ, показанной на рис. 3, $c$ пунктирной линией $\mathrm{D}$, полученной аналитически [19]. Однако для $\mathrm{S}_{31}$ видно, что набег фазы и эффективное волновое число начинают отличаться от ПМСВ, что связано с распространением сигнала по стороне магнитной полоски, к которой присоединена 

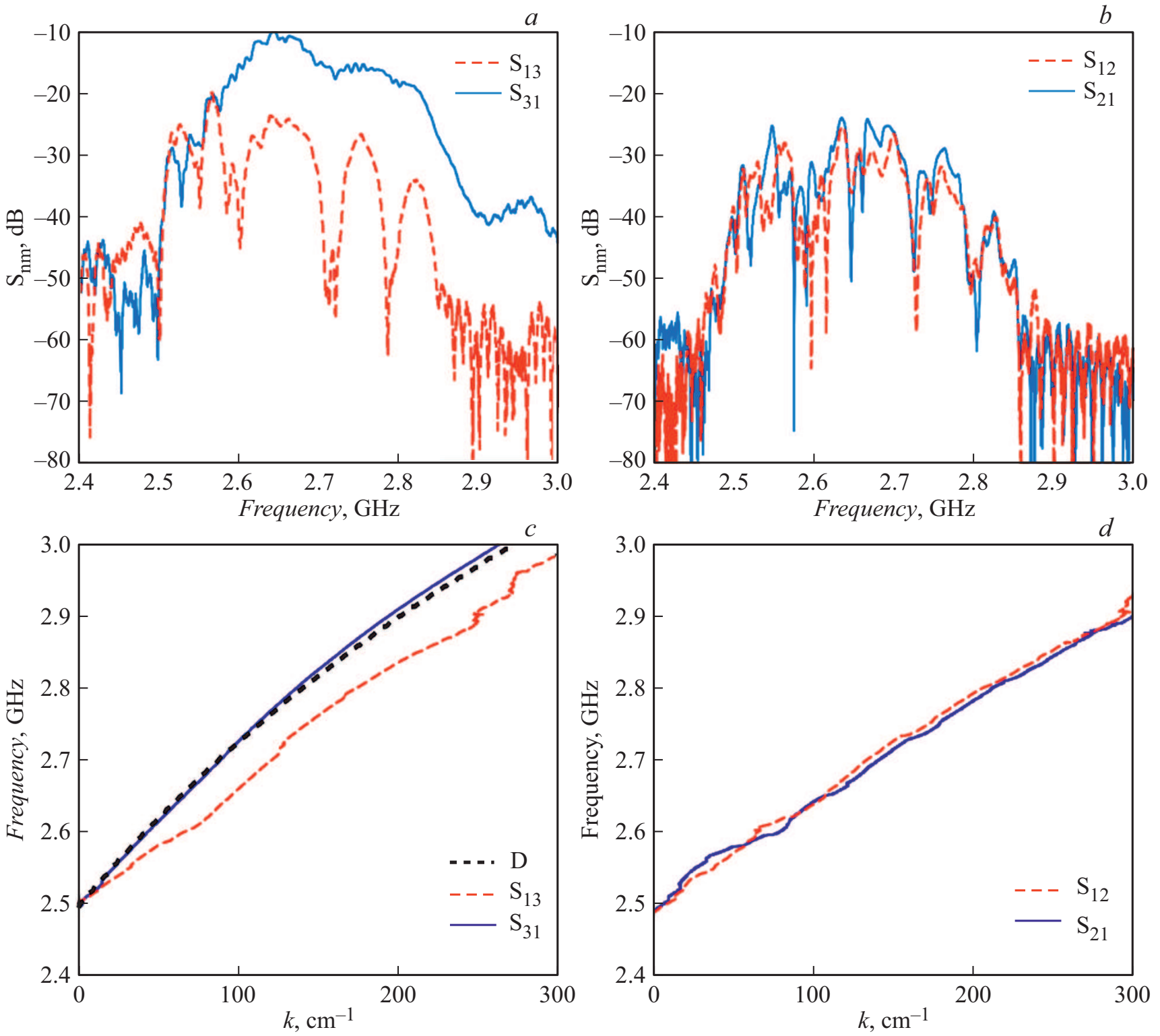

Рис. 3. Частотная зависимость модуля коэффициентов $\mathrm{S}_{n m}$ в секциях $\mathrm{S}_{1,3}(a)$ и $\mathrm{S}_{1,2}(b)$. Расчет эффективных волновых чисел $\mathrm{S}_{n m}$ в секциях $\mathrm{S}_{1,3}(c)$ и $\mathrm{S}_{1,2}(d)$.

вертикальная секция $\mathrm{S}_{2}$. Зависимость $k_{\mathrm{eff}}(f)$ для случая $\mathrm{S}_{12}$ и $\mathrm{S}_{21}$ также качественно совпадает с дисперсионной характеристикой ПМСВ (рис. $3, d)$.

\section{Заключение}

Таким образом, в работе исследован механизм управления передачи спин-волнового сигнала в трехмерном магнонном сплиттере. Используя методы микромагнитного моделирования для расчета равновесного распределения намагниченности, было показано однородное распределение поля при ортогональном сочленении магнонных микроволноводов. С помощью численного расчета характеристик спин-волнового транспорта было продемонстрировано, что в трехмерной структуре, образованной двумя магнитными полосками, возможна передача СВ в вертикальном направлении при Т-образном сочленении, что подтверждают экспериментальные результаты. Показано, что при изменении величины воздушного зазора между спин-волноведущими секциями оказывается возможным осуществлять пространственночастотную селекцию сигнала. С прикладной точки зрения, полученные результаты могут быть использованы для создания элементарного элемента межсоединения в многослойных системах обработки информации, использование которого позволит увеличивать плотность расположения функциональных элементов трехмерной магнонной сети. 


\section{Финансирование работы}

Работа выполнена при поддержке Минобрнауки России в рамках выполнения государственного задания (проект № FSRR-2020-0005).

\section{Конфликт интересов}

Авторы заявляют, что у них нет конфликта интересов.

\section{Список литературы}

[1] V.V. Kruglyak, S.O. Demokritov, D. Grundler. J. Phys. D, 43, 264001 (2010)

[2] A.V. Chumak, V.I. Vasyuchka, A.A. Serga, B. Hillebrands. Nature Phys., 11, 453 (2015).

[3] K. Roy, S. Bandyopadhyay, J. Atulasimha. Appl. Phys. Lett., 99, 063108 (2011).

[4] Yi-Pu. Wang, J.W. Rao, Y. Yang, Peng-Chao Xu, Y.S. Gui, B.M. Yao, J.Q. You, C.-M. Hu. Phys. Rev. Lett., 123, 127202 (2019).

[5] A.V. Sadovnikov, E.N. Beginin, S.E. Sheshukova, Yu.P. Sharaevskii, A.I. Stognij, N.N. Novitski, V.K. Sakharov, Yu.V. Khivintsev, S.A. Nikitov. Phys. Rev. B, 99, 054424 (2019).

[6] S. Neusser, D. Grundler. Adv. Mater., 21, 2927 (2009).

[7] S.A. Nikitov, A.R. Safin, D.V. Kalyabin, A.V. Sadovnikov, E.N. Beginin, M.V. Logunov, M.A. Morozova, S.A. Odintsov, S.A. Osokin, A.Yu. Sharaevskaya, Yu.P. Sharaevsky, A.I. Kirilyuk. Phys. Usp., 63, 945 (2020).

[8] D. Sander, S.O. Valenzuela, D. Makarov, C.H. Marrows, E.E. Fullerton, P. Fischer, J. McCord, P. Vavassori, S. Mangin, P. Pirro, B. Hillebrands, A.D. Kent, T. Jungwirth, O. Gutfleisch, C.G. Kim, A. Berger. J. Phys. D, 50, 363001. (2017).

[9] A.V. Sadovnikov, E.N. Beginin, S.E. Sheshukova, D.V. Romanenko, Y.P. Sharaevsky, S.A. Nikitov. Appl. Phys. Lett., 107, 202405 (2015).

[10] R. Perricone, X.S. Hu, J. Nahas, M. Niemier. Design of 3d nanomagnetic logic circuits: A full-adder case study, Design, Automation \& Test in Europe Conference \& Exhibition (DATE), 2014 10.7873/date.2014.132 (2014).

[11] A. Khitun, M. Bao, K.L. Wang. J. Phys. D, 43, 264005 (2010).

[12] M.T. Niemier, G.H. Bernstein, G. Csaba, A. Dingler, X.S. Hu, S. Kurtz, S. Liu, J. Nahas, W. Porod, M. Siddiq, E. Varga. J. Phys.: Condens. Matter., 23, 493202 (2011).

[13] E. Beginin, A. Sadovnikov, V. Sakharov, A. Stognij, Y. Khivintsev, S. Nikitov. J. Magn. Magn. Mater., 492, 165647 (2019).

[14] E.N. Beginin, A.V. Sadovnikov, A.Y. Sharaevskaya, A.I. Stognij, S.A. Nikitov. Appl. Phys. Lett., 112, 122404 (2018).

[15] G. Gubbiotti, A. Sadovnikov, E. Beginin, S. Nikitov, D. Wan, A. Gupta, S. Kundu, G. Talmelli, R. Carpenter, I. Asselberghs, I.P. Radu, C. Adelmann, F. Ciubotaru. Phys. Rev. Appl., 15, 014061 (2020).

[16] V.K. Sakharov, E.N. Beginin, Y.V. Khivintsev, A.V. Sadovnikov, A.I. Stognij, Y.A. Filimonov, S.A. Nikitov. Appl. Phys. Lett., 117, 022403 (2020).

[17] A. Vansteenkiste, J. Leliaert, M. Dvornik, M. Helsen, F. Garcia-Sanchez, B. Van Waeyenberge. AIP Adv., 4, 107133 (2014).

[18] G. Venkat, H. Fangohr. J. Magn. Magn. Mater., 34, 450 (2018).

[19] R. Damon, J.R. Eshbach. J. Phys. Chem. Solids, 19, 308 (1961). 